\title{
Manejo Forestal en el Siglo XXI
}

Forest management in the XXI Century

\author{
Oscar Alberto Aguirre-Calderón'
}

'Facultad de Ciencias Forestales. Universidad Autónoma de Nuevo León. Linares, Nuevo León, México. oscar.aguirrecl@uanl.edu.mx

\section{RESUMEN}

El manejo forestal sustentable en el siglo XXI es un principio que asegura la producción de diversos bienes y servicios a partir de los ecosistemas forestales de una manera perpetua y óptima, conservando siempre los valores de tales ecosistemas; es una estrategia de manejo de recursos naturales, en la cual las actividades forestales son consideradas en el contexto de las interacciones ecológicas, económicas y sociales, dentro de un área o región definida, a corto y largo plazo. El reto del manejo forestal sustentable es la gestión y utilización de los bosques y de los terrenos forestales de una manera y con una intensidad tales que conserven su diversidad biológica, su productividad, su capacidad de regeneración, su vitalidad y su capacidad de cumplir, en el presente y en el futuro, las funciones ecológicas, económicas y sociales pertinentes, a escala local, nacional y mundial, sin dañar otros ecosistemas. En este trabajo se revisa la evolución del concepto de manejo forestal y los nuevos paradigmas de su aplicación, destacando sus características como proceso administrativo, sus relaciones con la provisión de servicios ambientales, el papel de la certificación para el desarrollo del buen manejo forestal, la importancia del proceso de toma de decisiones, los retos del manejo forestal sustentable y la investigación necesaria para su ejecución con bases científicas. Se enfatiza asimismo la necesidad de formar recursos humanos, creando y mejorando capacidades para la planeación, ejecución y monitoreo del manejo forestal.

PALAbras Clave: servicios ambientales, certificación forestal, proceso analítico jerárquico.

\section{ABSTRACT}

The sustainable forest management in the twenty-first century is a principle that ensures the production of several goods and services from forest ecosystems in a permanent and optimal manner, maintaining always the values of such ecosystems; it is a strategy for natural resources management, in which forestry activities are considered in the ecological, economic and social context, within a defined area or region, in a short and long term. The challenge of sustainable forest management is the stewardship and use of the forests and forest lands in a manner and with an intensity that they preserve their biological diversity, its productivity, its capacity for regeneration, its vitality and ability to achieve, in the present and in the future, the ecological, economic and social functions relevant to local, national and global level, without causing damage to other ecosystems. This paper reviews the evolution of the forest management concept and the new paradigms for its implementation, highlighting their features such as administrative process, its relations with the provision of environmental services, the role of the certification for the development of good forest management, the importance of the decision-making process, the challenges of sustainable forest management, and the research needed for its implementation with scientific bases. It also emphasizes the need to train human resources, creating and improving capabilities for the planning, execution and monitoring of forest management.

KEYWORDS: environmental services, forest certification, analytic hierarchy process.

\section{INTRODUCCIÓN}

El manejo forestal comprende las decisiones y actividades encaminadas al aprovechamiento de los recursos forestales de manera ordenada, procurando satisfacer las necesidades de la sociedad actual, sin comprometer la provisión de bienes y servicios para las generaciones futuras.
Los bosques naturales se manejaron en el pasado principalmente para la producción de madera y energía (leña y carbón). El aprovechamiento se realizó en algunos casos en forma excesiva, lo que ocasionó consecuentemente fuertes presiones hacia su protección, provocando la pérdida de importantes superficies de bosques, selvas y 
matorrales. Las prácticas de aprovechamiento se desarrollaron principalmente como respuesta a la demanda del mercado nacional e internacional, básicamente de maderas, ignorando en muchos casos otros aspectos muy importantes de los ecosistemas forestales como los ambientales y ecológicos, los bienes y servicios que brindan (principalmente la captura de carbono y los servicios hidrológicos), así como las implicaciones sociales, económicas, ambientales e institucionales de los ecosistemas forestales y su manejo.

Desde hace algunas décadas, el manejo forestal considera en el proceso de toma de decisiones tres factores: el económico, el social y el ecológico, orientando la cosecha de productos o la provisión de servicios ambientales de acuerdo con las capacidades de los ecosistemas. Tiene entonces que ver con la sociedad y las personas, y la necesidad de que deban y puedan mantener y aumentar los servicios, beneficios económicos y la salud de los ecosistemas forestales para su desarrollo y mejor calidad de vida.

La tendencia en el siglo XXI es manejar el bosque en el marco de una visión ecosistémica, paisajista, integral, participativa y de uso múltiple, orientado a la obtención del rendimiento sostenido de los diversos productos, bienes y servicios que ofrece, con el fin de mejorar las condiciones y calidad de vida de la sociedad, dando origen al concepto de Manejo Forestal Sustentable o Manejo Forestal Sostenible (MFS). El manejo forestal sustentable moderno se concibe entonces como un sistema de toma de decisiones multiobjetivo que atiende los factores ecológico, económico y social. Lejos ha quedado el concepto de considerar como único bien aprovechable la madera y como indicador de buen manejo el minimizar los impactos ambientales de la cosecha.

El manejo forestal sustentable es un principio que asegura la producción de diversos bienes y servicios a partir de los ecosistemas forestales, de una manera perpetua y óptima, conservando siempre los valores de tales ecosistemas; es una estrategia de manejo de recursos naturales, en la cual las actividades forestales son consideradas en el contexto de las interacciones ecológicas, económicas y sociales, dentro de un área o región definida, a corto y largo plazo. El manejo forestal sustentable es entonces la gestión y utilización de los bosques y de los terrenos forestales de una manera y con una intensidad tales que conserven su diversidad biológica, su productividad, su capacidad de regeneración, su vitalidad y su capacidad de cumplir, en el presente y en el futuro, las funciones ecológicas, económicas y sociales pertinentes, a escala local, nacional y mundial, sin dañar otros ecosistemas.

\section{MANEJO FORESTAL COMO PROCESO ADMINISTRATIVO}

Los bosques han estado sujetos a intensas presiones por actividades humanas. El rápido cambio demográfico, los acelerados avances tecnológicos y el crecimiento de la demanda de energía han ejercido nuevas presiones a la actividad forestal para abordar problemas globales emergentes, particularmente energía y cambio climático. El crecimiento de la demanda de productos forestales y servicios está determinada por múltiples factores, y el crecimiento poblacional es una de las causas más importantes (Bettinger et al., 2009). El efecto demográfico por sí solo causará cambios significativos en la demanda de bienes y servicios provenientes del bosque. Los tomadores de decisiones del sector forestal se enfrentarán en el presente siglo al gran reto de buscar responder a las necesidades crecientes de productos forestales mientras se mantienen las múltiples funciones de los ecosistemas forestales.

El manejo forestal es esencialmente un proceso administrativo. Como tal, comprende entre otras las fases de organización, planeación, ejecución y control, teniendo como resultado el desarrollo futuro de los ecosistemas forestales (Knoke et al., 2012). En este marco hay tres cuestiones importantes a considerar: ¿cuáles son los objetivos que las actividades de manejo esperan alcanzar?; ¿cuáles son los procedimientos operativos y recursos financieros requeridos para ejecutar un plan de acción programado para lograr los objetivos? y ¿cuáles son los criterios que pueden ser empleados para evaluar la medida en la cual los objetivos son alcanzados? Los manejadores forestales deben establecer objetivos adecuados, tomar los pasos necesarios para alcanzar estos objetivos y medir el 
nivel de éxito en el cumplimiento de los mismos, esto es, realizar la planeación, la ejecución y el monitoreo del manejo del bosque.

El establecimiento de objetivos de manejo forestal es una tarea compleja que consiste en determinar los productos y servicios a ser obtenidos del bosque y especificar las medidas de logro de las metas asociadas. Tradicionalmente, los manejadores de bosques seleccionaban una combinación de producción de madera, generación de empleo y beneficios económicos como los principales componentes del programa de manejo forestal. En el presente siglo, establecer objetivos de manejo debe contemplar una visión multiobjetivo, teniendo en cuenta que muchos de ellos compiten entre sí y que no todas las metas pueden expresarse en unidades físicas, lo que dificulta su medición cuantitativa. Por otra parte, los objetivos deberán definirse para diferentes plazos y a diferentes escalas, desde el paisaje hasta la unidad mínima de manejo (Gadow et al., 2004).

La visión sobre cómo y para qué se debe manejar el bosque ha adquirido una nueva dimensión en el presente siglo. La planeación a largo plazo continuará sin duda teniendo su lugar en el manejo forestal, considerando que en esta nueva era las metas cambiantes caracterizarán las prácticas forestales, lo que dará vigencia al manejo adaptativo. El manejador forestal deberá ser perceptivo al ambiente institucional que rodea al bosque y a los valores culturales para reajustar los objetivos predeterminados en su plan de manejo a lo largo de un camino; el reto es lidiar con metas e hitos cambiantes en el manejo de bosques.

Los objetivos de la actividad forestal no solo varían a diferentes escalas, cambian también a través del tiempo y difieren de un contexto socioeconómico a otro. Las metas de manejo requieren ser revisadas constantemente, considerando los valores sociales prevalecientes, el estado de la tecnología disponible y el nivel de desarrollo económico, así como las percepciones públicas de las condiciones ecológicas generales del bosque y el impacto potencial de los regímenes de manejo propuestos (Lal, 2007). Además, el nivel de logro de los objetivos de manejo seleccionados se mide por un conjunto de criterios que se actualizan periódicamente.

\section{MANEJO FORESTAL Y SERVICIOS DE LOS ECOSISTEMAS}

En las últimas dos décadas se han observado cambios en la valoración social de los recursos naturales y la forma en que estos se manejan, lo que ha ocasionado un cambio fundamental en el contexto en el cual se desarrollan las actividades del manejo forestal (Aguirre, 1997). Los bosques no son más solamente una fuente de madera; en su lugar se convierten en fuente de una variedad de productos y servicios, incluyendo la diversidad biológica, hábitats, así como oportunidades recreativas y educativas.

En el siglo XXI los servicios que proveen los ecosistemas forestales son tanto o más importantes que los productos maderables. Por ello, los sistemas de manejo deben contemplar no solo los sistemas de cosecha sostenible, sino la provisión permanente de los servicios de los ecosistemas forestales. Lo anterior representa un reto para los manejadores forestales, a la vez que ofrece un amplio marco de oportunidades para la creatividad en cuanto a desarrollo de modelos de manejo y de estrategias de gestión de los recursos bajo su responsabilidad, internalizando el valor del bosque en la conservación de la biodiversidad, provisión de servicios hidrológicos y captura de carbono, básicamente.

Las evidencias de los beneficios ecológicos, económicos y sociales de los nuevos modelos de manejo dejan claro que no se trata de optar entre desarrollo o conservación, sino que existen respuestas posibles desde las personas que habitan los bosques para cumplir los objetivos del desarrollo sostenible en las regiones forestales. Esta es la base de los nuevos paradigmas del manejo forestal sustentable (Higman et al., 2000; Hawksworth y Bull, 2006; Poff, 2008; Grossberg, 2009; Malmsheimer et al., 2009).

Las oportunidades de desarrollo del manejo forestal sostenible se ubican también en las estrategias de gestión de recursos forestales que la comunidad internacional ha desarrollado en los últimos años, entre las que destaca REDD+. En 2005, un grupo de países llevó el tema de la 
deforestación evitada a la agenda de la Conferencia de las Partes (COP), realizada en Montreal (COP 11). Durante la COP 13, en Bali en 2007, se reconoció la reducción de emisiones por deforestación y degradación de los bosques (REDD) como un mecanismo válido en la lucha contra el cambio climático. Inicialmente, las discusiones se centraron en lograr el reconocimiento del potencial de la reducción de emisiones causadas por deforestación (RED); la segunda $\mathrm{D}$ se incluyó más tarde al reconocer que la degradación de los bosques representa también una fuente importante de emisiones de gases de efecto invernadero, definiéndose el término REDD. Se incorporó luego el papel de la conservación, el manejo sustentable de los bosques y el mejoramiento de los almacenes de carbono, elementos que constituyen el signo +. REDD+ denota entonces las actividades que reducen las emisiones por evitar la deforestación y degradación forestal y contribuyen a la conservación, manejo sostenible de los bosques y mejoramiento de los acervos de carbono forestal, y que tienen el potencial de generar significativos beneficios sociales y ambientales.

La implementación de REDD+ representa una de las estrategias más importantes de gestión de los bosques del futuro y aunque enfrenta retos técnicos y prácticos en lo que a su implementación se refiere, diversos países muestran avances importantes en la fase de preparación y desarrollo de proyectos a nivel local o regional (Calmel et al., 2010). Durante la COP 16 se presentó la Visión de México sobre REDD+ (SEMARNAT-CONAFOR, 2010) y el país muestra avances en la implementación de acciones tempranas. Una acción temprana REDD+ es un esfuerzo articulado institucionalmente a nivel subnacional (regional y local) que permite atender las causas de la pérdida de bosques y del carbono forestal mediante diferentes instrumentos de política pública que generen oportunidades para el desarrollo para los habitantes de los bosques.

A través de las acciones tempranas REDD+ se busca, a escala local, la promoción de la competitividad en las diferentes actividades productivas, incluyendo las actividades agropecuarias asociadas al bosque; el fortalecimiento del manejo comunitario de los bosques y de sus empresas forestales; la diversificación productiva, y la conservación y protección de los bosques, de sus servicios y su biodiversidad en el largo plazo. La implementación de proyectos de esta naturaleza es una alternativa factible a corto plazo.

\section{CERTIFICACIÓN DEL MANEJO FORESTAL}

El monitoreo es una fase determinante en el proceso administrativo denominado manejo forestal y cobrará cada vez mayor importancia en el futuro. El manejo forestal sostenible puede ser caracterizado y evaluado a través de principios, criterios e indicadores. Diversas instituciones han desarrollado con detalle estos aspectos como base para evaluar la sustentabilidad según el tipo de ecosistema forestal, escala de manejo y productos aprovechados. Lo anterior ha dado origen a la certificación forestal, que constituye un proceso que evalúa el desempeño del manejo forestal, para garantizar que los productos que provienen de los ecosistemas forestales tomen en cuenta estándares que sean: ambientalmente adecuados, económicamente viables y socialmente benéficos.

Los principios actuales del manejo forestal sustentable reconocidos internacionalmente son (Gadow et al., 2000; Davis et al., 2001):

- Tiende hacia el uso sustentable de los recursos de los ecosistemas.

- Es holístico.

- Está basado en el ecosistema.

- Tiene una perspectiva de paisaje.

- Establece objetivos múltiples.

- Es integrador.

- Incluye la participación.

- Se basa en el monitoreo.

- Es adaptativo.

- Está basado en ciencia adecuada y buen juicio.

- Toma en cuenta reacciones cognocitivas, emocionales y morales.

- Está basado en principios precautorios.

Bajo esta nueva visión se han gestado a nivel internacional y nacional instrumentos de promoción del manejo 
forestal sustentable que se expresan en estándares de certificación, los cuales integran principios, criterios e indicadores de buen manejo que norman cómo debe realizarse la gestión de los recursos forestales. Los principios de manejo forestal sustentable del denominado Proceso de Montreal, son:

1. Conservación de la diversidad biológica.

2. Mantenimiento de la capacidad productiva de los ecosistemas forestales.

3. Mantenimiento de la salud y vitalidad de los ecosistemas forestales.

4. Conservación y mantenimiento de los recursos suelo y agua.

5. Mantenimiento de la contribución de los bosques al ciclo global del carbono.

6. Mantenimiento y mejoramiento de los múltiples beneficios socio-económicos de largo plazo para satisfacer las necesidades de la sociedad, y

7. Creación de marcos legales, institucionales y económicos para la conservación y el manejo forestal sustentable.

A nivel nacional se han desarrollado también estándares de buen manejo forestal. En el caso de México, por ejemplo, el estándar se describe en la Norma Mexicana NMX-AA-143-SCFI-2008 para la certificación del manejo sustentable de bosque (Secretaría de Economía, 2008), cuyos principios son:

1. La Empresa o predio Forestal está legalmente constituida.

2. La Empresa o predio Forestal cuenta con una administración y archivos de registros de entradas y salidas de materias primas forestales.

3. La Empresa o predio Forestal muestra un compromiso a favor de la conservación del ecosistema forestal y mantiene una conducta apegada a la normatividad vigente.

4. La Empresa o predio Forestal observa la normatividad y realiza acciones para prevenir y mitigar efectos adversos ocasionados por el aprovechamiento forestal.
5. La Empresa o predio forestal lleva registros y aplica procedimientos que aseguran la verificación de volúmenes y de la legal procedencia de la materia prima forestal.

6. La Empresa o predio Forestal proporciona condiciones laborales adecuadas a los trabajadores.

7. La Empresa o predio Forestal mantiene relaciones de respeto y cooperación con las comunidades locales dentro de, o adyacentes al predio bajo manejo forestal.

8. La Empresa o predio Forestal tiene establecido un procedimiento de monitoreo y evaluación de los impactos a la vegetación, fauna, calidad del agua y el suelo.

9. La Empresa o predio Forestal incorpora aspectos socioeconómicos que coadyuvan con el manejo sustentable del recurso forestal.

La certificación de buen manejo forestal pretende destacar situaciones en las cuales el concepto de MFS está bien aplicado en la práctica, viéndose reflejado tanto en las condiciones en que se encuentran los ecosistemas como en los beneficios que generan a sus propietarios y a la sociedad en general, para que sean empleados como modelos de referencia en otros bosques de condiciones y contextos semejantes. La certificación es un marco regulatorio para hacer operativos los conceptos modernos del enfoque de ecosistemas y el manejo forestal sustentable.

La certificación es un componente de la tendencia hacia modelos de manejo forestal de amplia visión y mayor participación, los cuales se agrupan bajo el concepto general de enfoque de ecosistemas, y ha sido también un instrumento para el desarrollo de mejores prácticas de manejo, que orientan el quehacer de los responsables de la gestión de los recursos forestales. Guías de mejores prácticas de manejo forestal se han sistematizado para diferentes regiones y escalas territoriales, en México, por ejemplo, el manual de mejores prácticas de manejo forestal para la conservación de la biodiversidad en ecosistemas templados de la región norte incluye (CONAFOR-PNUD, 2012): 
Prácticas a nivel paisaje:

- Conectividad de hábitats.

- Claros en el bosque.

- Red de áreas de conservación.

- Protección de hábitats en las partes altas de las cuencas.

- Protección de ecosistemas ribereños.

- Bosques sobremaduros.

Prácticas a nivel rodal:

- Estructura vertical y horizontal.

- Estructura y diversidad de especies.

- Formación de micro-hábitats.

- Actividades recreativas.

- Biomasa residual de la cosecha forestal.

Prácticas a nivel sitio:

- Protección de áreas de importancia crítica.

- Mantenimiento de árboles secos o con cavidades.

- Limpieza de las áreas de corta.

Los sistemas de certificación exitosos se han sido asociado a enfoques plurales y participativos para la toma de decisiones en los bosques y han ido más allá de simplemente mejorar las técnicas de cosecha, abordando aspectos sociales y ambientales. La certificación ha contribuido también a elevar el conocimiento y comprensión del manejo forestal sustentable. Los criterios e indicadores han propiciado un debate público sobre los bosques más estructurado y han resultado en una más amplia aceptación de la aplicación del manejo forestal como estrategia de conservación del bosque.

\section{TOMA DE DECISIONES}

La función del manejo forestal es administrar la capacidad productiva del bosque para generar diversos de bienes y servicios para satisfacer necesidades humanas. El final de la primacía de la madera hacia finales del siglo pasado marca el inicio de una nueva era en la que los planes de manejo deben ser hechos a nivel de paisaje con el propósito de mantener una diversidad de funciones de los ecosis- temas (Hussain, 2012). El cambio de la planeación forestal de nivel rodal a nivel paisaje amplía la visión de los objetivos de manejo forestal para abarcar el papel de los bosques en proveer ingresos y oportunidades de desarrollo, preservar la diversidad biológica y los hábitats, y posibilitar un apropiado funcionamiento del sistema global del carbono y del ciclo hidrológico (Sayer et al., 2007). Los esquemas de toma de decisiones han cambiado en consecuencia integrando actualmente consideraciones económicas, ambientales, sociales y culturales, con lo que el proceso se ha tornado más complejo. Prácticamente en todo el mundo se ha discutido quién toma o debe tomar las decisiones acerca del bosque y cómo se toman o debieran tomarse tales decisiones.

Dado que el manejo debe realizarse con una visión multiobjetivo, para el diseño de planes de manejo en el siglo XXI se aplicarán en mayor medida técnicas cuantitativas de toma de decisiones que permitan optimizar objetivos bajo una serie de restricciones. En este marco, las técnicas de investigación de operaciones, particularmente la programación lineal, posibilitan la planeación del manejo forestal a partir de la determinación de un objetivo de manejo y del establecimiento de una serie de restricciones (Pukkala, 2002; Buongiorno y Gilles, 2003; Herat y Prato, 2006; Kaiser y Messer, 2011). Un modelo de programación lineal tiene la estructura siguiente:

$$
\operatorname{Max} Z=c_{1} X_{1}+c_{2} X_{2}+\ldots+c_{n} X_{n}
$$

sujeto a:

$$
\begin{aligned}
& a_{11} X_{1}+a_{12} X_{2}+\ldots a_{1 n} X_{n}=b_{1} \\
& a_{21} X_{1}+a_{22} X_{2}+\ldots a_{2 n} X_{n} \geq b_{2} \\
& \ldots \\
& a_{m 1} X_{1}+a_{m 2} X_{2}+\ldots a_{m n} X_{n} \leq b_{m}
\end{aligned}
$$

Max Z representa la función objetivo para cada opción de manejo forestal, por ejemplo utilidades netas; el resto de las ecuaciones constituyen restricciones de igualdad, mínimo y máximo que incorporan los recursos disponibles para el desarrollo de la opción de manejo propuesta; 
expresan asimismo el resto de los objetivos que se proponen lograr con la aplicación del modelo. El objetivo a maximizar puede ser la cosecha de un bien determinado, las restricciones pueden expresar mantenimiento de la biodiversidad, un nivel mínimo de cobertura residual permisible y un nivel determinado de volumen en pie postcosecha.

Por otra parte, la formulación y selección de alternativas de manejo forestal se podrá realizar incorporando herramientas de toma de decisiones como la denominada Proceso Analítico Jerárquico, que posibilita la participación de los sectores sociales involucrados.

El proceso analítico jerárquico es una técnica para la toma de decisiones con atributos múltiples (Saaty, 2006, 2008). El método consiste en formalizar la comprensión intuitiva de problemas complejos mediante la construcción de un modelo jerárquico. El propósito del método es permitir que quienes deben tomar decisiones puedan estructurar un problema multicriterio en forma visual, mediante la construcción de un modelo que básicamente contiene tres niveles: meta u objetivo, criterios y alternativas.

Una vez construido el modelo jerárquico, se realizan comparaciones por pares entre dichos elementos (criterios-subcriterios y alternativas) y se atribuyen valores numéricos a las preferencias señaladas por las personas, entregando una síntesis de las mismas mediante la agregación de esos juicios parciales. El proceso analítico jerár- quico permite dar valores numéricos a los juicios dados por las personas, logrando medir cómo contribuye cada elemento de la jerarquía al nivel inmediatamente superior del cual se desprende. Para estas comparaciones se utilizan escalas de razón en términos de preferencia, importancia o probabilidad. Para un objetivo dado, las comparaciones se realizan por pares de elementos, primero planteando la pregunta: ¿Cuál de los dos es el más importante? y segundo: ¿Por cuánto?

El proceso analítico jerárquico se ajusta bien al tipo de problemas complejos de toma de decisiones que involucran metas múltiples relacionadas con la planeación del manejo forestal (Schmoldt et al., 2001; Pérez-Rodríguez et al., 2012). Para ilustrar la aplicación del método en la toma de decisiones en manejo forestal se modelizó un ejemplo en el que el objetivo global es la obtención de la Mejor Estrategia de Manejo (Aguirre et al., 2013). En un primer nivel jerárquico, los criterios de decisión son: Producción Maderable, Estructura del Subrodal y Provisión de Servicios Ambientales. El segundo nivel jerárquico de criterios subordina el Valor de la Cosecha y el Valor de las Existencias Residuales a la Producción Maderable; la Diversidad de Especies y la Diversidad Dimensional a la Estructura del Subrodal y, finalmente, los Servicios Hidrológicos y la Captura de carbono a la Provisión de Servicios Ambientales. Las alternativas de manejo son Aclareo Ligero, Aclareo Moderado y No Cosechar (Fig. 1).

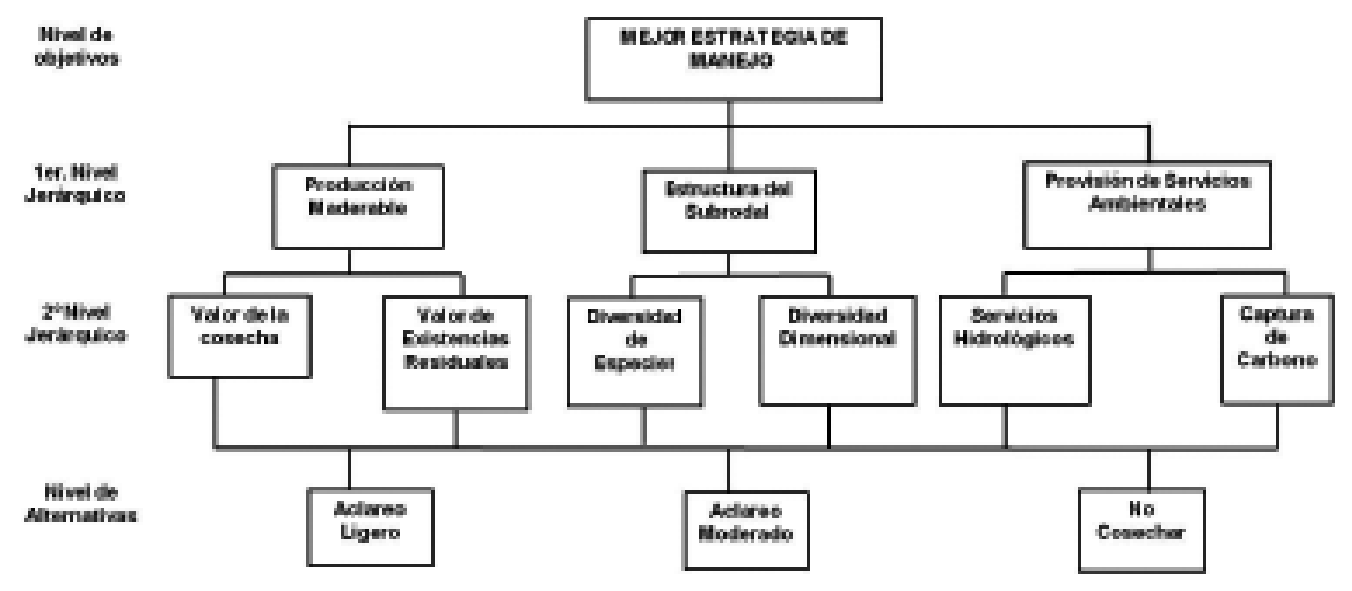

Figura 1. Proceso Analítico Jerárquico.

Representación del objetivo y estructura de criterios de valoración y alternativas de manejo. 
La elección de alternativas de manejo mediante el proceso analítico jerárquico se realiza empleando software desarrollado específicamente para este objeto. Entre los recursos libres disponibles en la Web destaca el programa HIPRE 3+ desarrollado por Mustajoki y Hämäläinen (2000).

\section{RETOS DEL MANEJO FORESTAL}

El manejo forestal es una experiencia gratificante para quienes son responsables de su ejecución, ya que enfrenta retos en de diversas áreas. Existen numerosos retos económicos, dentro de los cuales destacan diversas necesidades como son: generar beneficios, no operar con pérdidas, operar dentro de un presupuesto y generar ingresos o retornos financieros competitivos en comparación con otras inversiones. Estos retos económicos usualmente se expresan en unidades monetarias por lo que es necesario internalizar en las valoraciones las externalidades de los servicios de los ecosistemas.

Hay asimismo numerosos retos ambientales, relacionados con el mantenimiento y desarrollo del hábitat para la vida silvestre, la calidad del agua, calidad del suelo, calidad del aire y diversidad biológica, muchos de los cuales están contemplados en leyes y regulaciones pero que son difíciles de llevar a la práctica en muchas situaciones.

No menos importantes son los retos sociales respecto al manejo forestal. Convencer a la opinión pública de que el bosque está siendo manejado responsablemente no es un asunto trivial, de ahí la relevancia de la aplicación de instrumentos de política forestal como la certificación forestal (leyes y regulaciones) que guíen el manejo de los recursos forestales. Existe también la necesidad de generar empleos para las comunidades locales y pagar estos empleos con salarios suficientes.

Los retos tecnológicos relacionados con el manejo forestal son de muy diversa índole, lo mismo que los relacionados con los procesos de planeación. Otros retos del manejo forestal se relacionan con los sistemas silvícolas y operaciones forestales (cosecha, reducción de combustibles, etc.) que posibiliten el mantenimiento de la diversidad biológica y el mantenimiento de la condición de los ecosistemas. El manejo forestal es una actividad de largo plazo, lo que da como resultado que los productos del manejo están sujetos a riesgos potenciales por las condiciones ambientales, así como por la propia influencia antropogénica. Lo anterior representa también un importante reto, ya que el desarrollo de planes de manejo para áreas boscosas tiene que integrar estas incertidumbres, las cuales pueden ser numerosas para planes de acción que comprenden grandes superficies y largos periodos. El riesgo no puede ser eliminado ni totalmente cuantificado al manejar sistemas de recursos naturales y la percepción del mismo cambia con el tiempo; la tarea de los manejadores de recursos naturales es realizar buenos planes y ejecutar un buen manejo basados en la evaluación de los riesgos involucrados.

Debe reconocerse que los procesos de planeación y toma de decisiones se ven frecuentemente obstaculizados por retos internos en una organización. Estos incluyen limitaciones tecnológicas (sistemas computacionales obsoletos, programas de software inadecuado etc.), diferencias de visión entre los miembros, carencia de datos y falta de apoyo de un equipo de manejo organizacional. La tecnología puede ser tan obsoleta que se convierte en cuello de botella del proceso de planeación y superar este reto puede requerir planeación en sí misma a fin de lograr una mejor toma de decisiones.

En muchos procesos de planeación forestal, la obtención y procesamiento de información puede tomar más de la mitad del tiempo invertido en el proceso de planeación. Contar con un sistema de información geográfica, bases de datos suficientes, proyecciones de crecimiento e incremento para cada prescripción de manejo, precios, costos, medidas de la calidad potencial del hábitat y niveles de las restricciones que serán aplicadas representa también un importante reto. Colectar, manejar, corregir y dar formato a los datos se lleva a cabo generalmente por varias personas en la organización, y es, desafortunadamente, uno de los aspectos poco valorados en el manejo. La motivación de las personas para capacitarse y apoyar el proceso de planeación es asimismo un reto, al igual que una serie contratiempos que pueden ocurrir durante el desa- 
rrollo del plan; sin embargo, muchos de los retos de la planeación que son internos a una organización de recursos naturales pueden ser superados si se reconocen y discuten entre sus miembros.

\section{LA INVESTIGACIÓN NECESARIA}

Para la planeación, ejecución y monitoreo del manejo forestal sustentable se requiere de la generación de conocimiento científico como apoyo a la toma de decisiones y medición del logro de los objetivos establecidos. La producción maderable será el principal objetivo en muchas regiones, y para su implementación es necesario conocer la dinámica de los ecosistemas bajo manejo a fin de garantizar una cosecha sustentable. A pesar de que el manejo forestal maderable ha sido fundamental en el pasado y existen numerosas investigaciones sobre el desarrollo de las especies en diversas regiones, las investigaciones sobre volumen, crecimiento, incremento, densidad y cosecha permisible, entre otras, siguen siendo necesarias y vigentes (Pretzsch, 2009), a la par de las referentes al conocimiento de las interacciones de los diversos elementos de los ecosistemas y de estos con la sociedad y otros ecosistemas.

Dado el cambio en el manejo silvícola de ser principalmente enfocado a bosques regulares hacia un sistema de manejo enfocado a bosques mixtos incoetáneos a pequeña escala y/o a árboles individuales, las tablas de producción se tornarán gradualmente poco confiables. Como una alternativa potencial, será necesario continuar el desarrollo de modelos de crecimiento para pronosticar el crecimiento de los árboles dentro de un rodal atendiendo condiciones de edad, mezcla de especies y manejo silvícola, posibilitando la flexibilidad necesaria para modelizar bosques bajo manejo (Hasenauer, 2006).

El siglo XX se caracterizó por el uso de petroquímicos para obtención de energía y generación de productos. La creciente preocupación acerca del impacto de las emisiones de gases de efecto invernadero en el cambio climático ha resultado en un incremento de oportunidades para energías alternativas, incluida la bioenergía de las fibras de madera (Bravo et al., 2008). En el presente, la industria de productos forestales juega un papel determinante en el sector forestal, y las actividades de manejo se ven afectadas por la fortaleza o debilidad de los precios de mercado de productos forestales. Sin embargo, los factores que determinan de qué manera los bosques deben ser manejados están cambiando rápidamente. Es claro que el siglo XXI va a ser caracterizado por una bioeconomía, donde los cultivos de biomasa, incluyendo fibras de madera, proveerán energía renovable, productos químicos y materiales. Obviamente, se requiere investigación para examinar la factibilidad técnica y económica, así como las implicaciones ecológicas de usar biomasa forestal como alternativa a los combustibles fósiles.

Actualmente se reconoce la importancia de los bosques como un medio para mitigar las emisiones de gases efecto invernadero, considerados los causantes del cambio climático, particularmente el bióxido de carbono $\left(\mathrm{CO}_{2}\right)$ es el gas con mayor participación y se fija a través del proceso de fotosíntesis, en el que las plantas utilizan $\mathrm{CO}_{2}$ y liberan oxígeno. Así, para contrarrestar las emisiones de carbono, un número creciente de instituciones y organizaciones de diferentes países están desarrollando planes para preservar bosques naturales, mejorar el manejo de bosques y establecer plantaciones forestales, ya que los árboles almacenan el carbono por periodos prolongados. En este marco, la investigación necesaria refiere al desarrollo de métodos para la evaluación de la capacidad de captura de carbono en ecosistemas forestales, con objeto de valorar este servicio ambiental con procedimientos confiables que sean costo-eficientes (Husch et al., 2003; Ravindranath y Ostwald, 2007; Van Laar y Akça, 2007; Hoover, 2008; Bigsby, 2009). En México, a pesar de que se ha observado un incremento notable en el desarrollo de metodologías de evaluación para estimar la biomasa y el carbono contenido en diferentes ecosistemas forestales (De Jong et al. 1995; Ordóñez et al., 2001; Pimienta et al., 2007; Rodríguez-Laguna et al., 2008; Domínguez-Cabrera et al., 2009; Figueroa-Navarro et al., 2010, Aguirre y Jiménez, 2011), aún se requiere de investigaciones en numerosas especies y ecosistemas para apoyar el desarrollo de proyectos como REDD+ que contribuyan a un buen manejo forestal. 
La investigación es necesaria asimismo para generar mejores esquemas de toma de decisiones y para la valoración económica de las alternativas de manejo propuestas, donde deben internalizarse las contribuciones de los bienes y servicios "no de mercado" que proveen los ecosistemas forestales.

Los aspectos de investigación mencionados sn apenas algunos de los que deberán abordarse para el desarrollo de un mejor manejo forestal. El enfoque dinámico del manejo de los ecosistemas forestales demandará nuevos retos en la investigación y generación de conocimientos, que permitan a los responsables de la gestión de estos recursos el mejoramiento y la creación de capacidades para el mejor desempeño de las tareas de planeación, ejecución y monitoreo del manejo forestal.

El avance en el desarrollo de los diferentes tópicos de investigación mencionados, posibilitará la integración de sistemas de apoyo a la toma de decisiones (DSS por sus iniciales en ingles), cuyo diseño deberá realizarse por equipos interdiciplinarios y que puedan ser aplicados en un amplio rango de condiciones ecológicas, sociales y económicas; tengan costos accesibles; sean de fácil comprensión y operación; sean interactivos; contengan interfaces amigables que expliquen los impactos de las alternativas de manejo; faciliten la producción de documentos para las instituciones regulatorias; proporcionen información social, económica y ambiental de las áreas de manejo y posibiliten la implementación de modelos cuantitativos para la toma de decisiones (Moreno-Sánchez y TorresRojo, 2010).

\section{COMENTARIOS FINALES}

En el siglo XXI, el manejo forestal deberá seguir avanzando hacia la sustentabilidad, teniendo en mente que esta meta es alcanzable a diferentes escalas, por diferentes actores y mediante diferentes estrategias, herramientas y prácticas; no existe un modelo único.

A pesar de los avances en la fase de planeación del manejo forestal sustentable, es necesario mejorar la ejecución del mismo. Para ello se requerirá de recursos humanos operativos con capacidades certificadas, amplia visión del manejo y voluntad de interacción en grupos interdisciplinarios. Los responsables del manejo deberán mejorar sus capacidades no solamente en aspectos técnicos, sino para el fortalecimiento del capital social que posibilite una adecuada organización y gestión empresarial.

El manejo forestal sustentable constituye una práctica de gestión de los recursos forestales que asegura los procesos y funciones del bosque, posibilita la conservación de la biodiversidad y satisface necesidades sociales. En el siglo XXI la comunidad internacional seguirá adecuando respuestas a las preguntas: manejo forestal ¿para qué? y ¿para quién?

\section{REFERENCIAS}

Aguirre C., O.A. 1997. Hacia el manejo de ecosistemas forestales. Madera y Bosques 3(2):3-11.

Aguirre-Calderón, O.A. y J. Jiménez-Pérez. 2011. Evaluación del contenido de carbono en bosques del Sur de Nuevo León. Revista Mexicana de Ciencias Forestales 2(6):73:83.

Aguirre-Calderón, O.A., J. Jiménez-Pérez, E. Alanís-Rodríguez e I. Yerena-Yamallel. 2013. Manejo Forestal. In: C. Cantú A., M. Rovalo M., J. Marmolejo M., S. Ortiz H. y F. Seriñá G., eds. Historia Natural del Parque Nacional Cumbres de Monterrey, México. UANL-CONANP. México. p:275-286.

Bettinger, P., K. Boston, J.P. Siry y D.L. Grebner. 2009. Forest Management and Planning. Elsevier. Amsterdam. 331 p.

Bigsby, H. 2009. Carbon banking; creating flexibility for forest owners. Forest Ecology and Management 257(1):378383.

Bravo, F., V. LeMay, R. Jandl y K. Gadow. 2008. Managing Forest Ecosystems: The Challenge of Climate Change. Springer. Dordrecht. 338 p.

Buongiorno, J. y J.K. Gilles. 2003. Decision methods for forest resource management. Academic Press. Amsterdam. 439 p.

Calmel, M., A. Martinet, N. Grondard, T. Dufour, M. Rageade y A. Ferté-Devin. 2010. REDD+ at proyect scale. Evaluation and development guide. ONF International. 215 p.

CONAFOR-PNUD. 2012: Manual de mejores prácticas de manejo forestal para la conservación de la biodiversidad 
en ecosistemas templados de la región norte de México. $76 \mathrm{p}$.

Davis, L.S., K.N. Johnson, P. Bettinger y T.E. Howard. 2001. Forest manegement. to sustain ecological, economic and social values. 4a ed. Waveland Press, Inc. Long Grove. 804 p.

De Jong, B.H.J., G. Montoya-Gómez, K. Nelson, L. Soto-Pinto y R. Tipper. 1995. Community forest management and carbon sequestration: A feasibility study from Chiapas, Mexico. Interciencia 20(6):409-416.

Domínguez-Cabrera, G., O.A. Aguirre-Calderón, J. JiménezPérez, R. Rodríguez-Laguna y J.A. Díaz-Balderas. 2009. Biomasa aérea y factores de expansión de especies arbóreas del sur de Nuevo León. Revista Chapingo Serie Ciencias Forestales y del Ambiente 15(1):59-64.

Figueroa-Navarro, C.M., G. Ángeles-Pérez, A. Velázquez-Martínez y H.M. de los Santos-Posadas. 2010. Revista Mexicana de Ciencias Forestales 1(1):105-112.

Gadow, K., T. Pukkala y M. Tomé. 2000. Sustainable forest management. Managing forest ecosystems. Kluwer Academic Publishers. Dordrecht. 356 p.

Gadow, K., S. Sánchez-Orois y O.A. Aguirre-Calderón. 2004. Manejo forestal con bases científicas. Madera y Bosques 10(2):3-16.

Grossberg, S.P. 2009. Forest management. Nova Science Publishers, Inc. Nueva York. 329 p.

Hasenauer, H. 2006. Sustainable forest management. Growth Models for Europe. Springer. Berlin. 398 p.

Hawksworth, D.L. y A.T. Bull. 2006. Forest diversity and management. Springer. Dordrecht. 547 p.

Herath, G. y T. Prato. 2006. Using multi-critera decision analysis in natural resource management. Ashgate. Hampshire. 239 p.

Higman, S., S. Bass, N. Judd, J. Mayers y R. Nussbaum. 2000. The sustainable forestry handbook. A practical guide for tropical forest managers on implementing new standards. Earthscan Pub. Londres. 289 p.

Hoover, C.M. 2008. Field measurements for forest carbon monitoring: a landscape-scale approach. Springer. Dordrecht. 258 p.
Husch, B., T.W. Beers y J.A. Kershaw. 2003. Forest mensuration. 4a ed. John Wiley \& Sons. Hoboken. 443 p.

Hussain A. 2012. Forest management: Innovative Practices. The Icfai University Press. Agartala. 163 p.

Kaiser; H.M. y K.D. Messer. 2011. Mathematical programming for agricultural, environmental, and resource economics. John Wiley \& Sons, Inc. Danvers. 494 p.

Knoke, T., T. Schneider, A. Hahn, V.C. Grieb y J. Röbiger. 2012. Forstbetriebsplanung als Entscheidungshilfe. Ulmer. Stuttgart. 408 p.

Lal, J.B. 2007. Forest Management: Classical Approach \& Current Imperatives. Natraj Publishers. Dehradun. 289 p.

Malmsheimer, R.W., P. Heffernan, S. Brink, D. Crandall, F. Deneke, C. Galik, E. Gee, J.A. Helms, N. McClure, M. Mortimer, S. Ruddell, M. Smith y J. Stewart. 2009. Forest management solutions for mitigating climate change in the United States. Society of American Foresters. Bethesda.137p.

Mustajoki J. y P. Hämäläinen. 2000. Web-HIPRE: Global decision support by value tree and AHP analysis. INFOR 38(3):208-220.

Moreno-Sánchez, R. y J.M. Torres Rojo. 2010. Forest management in Mexico: their characteristics and context for their creation and evolution. In: B. Manos, N. Matsatsinis, K. Paparrizos y J. Papathanasiou. Decision support systems in agriculture, food and the environment: trends, applications and advances. Information Science Reference. Hershey. p:74-100.

Ordóñez, J.A., B.H.J. de Jong y O. Masera. 2001. Almacenamiento de carbono en un bosque de Pinus pseudostrobus en Nuevo San Juan, Michoacán. Madera y Bosques $7(2): 27-47$.

Pérez-Rodríguez, F., B. Vargas-Larreta, O.A. Aguirre-Calderón, J.J. Corral-Rivas y A. Rojo-Alboreca. 2012. Proceso analítico jerárquico para seleccionar métodos de manejo forestal en Durango. Revista Mexicana de Ciencias Forestales 4(15):55-72.

Pimienta de la T., D.J., G. Domínguez C., O. Aguirre C., F.J. Hernández y J. Jiménez P. 2007. Estimación de biomasa y contenido de carbono de Pinus cooperi Blanco en Pueblo Nuevo, Durango. Madera y Bosques 13(1):35-46. 
Poff, B. 2008. Forest management in a spatio-temporal multiobjective decision-making framework. Verlag Dr. Muller. Saarbrücken.100 p.

Pretzsch, H. 2009. Forest dynamics, growth and yield. Springer. Dordrecht. 664 p.

Pukkala, T. 2002. Multi-objective forest planning. managing Forest Ecosystems. Kluwer Academic Publishers. Dordrecht. 207 p.

Ravindranath, N.H. y M. Ostwald 2007. Carbon inventory methods. Springer. Dordrecht. 308 p.

Rodríguez-Laguna, R., J. Jiménez-Pérez, J. Meza- Rangel, O. Aguirre-Calderón y R. Razo-Zárate 2008. Carbono contenido en un bosque tropical subcaducifolio en la reserva de la biósfera el cielo, Tamaulipas, México. Revista Latinoamericana de Recursos Naturales 4(2):215-222.

Saaty, T.L. 2006. Fundamentals of decisión making and priority theory with the analytic hierarchy process. $2 \mathrm{a}$ ed. RWS Publications. Pittsburgh. 478 p.

Saaty, T.L. 2008. The analytic hierarchy process for decisions in a complex world. RWS Publications. Pittsburgh. 343 p.
Sayer J., S. Maginnis y M. Laurie, eds. 2007. Forests in landscapes: ecosystem approaches to sustainability. The Earthscan Forest Library. Routledge. Sterling. 257 p.

Schmoldt, D.L., J. Kangas, G.A. Mendoza y M. Pesonen. 2001. The analytic hierarchy process in natural resource and environmental decision making. Managing forest ecosystems. Kluwer Academic Publishers. Dordrecht. 305 p.

Secretaría de Economía. 2008. Norma Mexicana NMX-AA143-SCFI-2008 para la certificación del manejo sustentable de bosque. $22 \mathrm{p}$.

SEMARNAT-CONAFOR. 2010. Visión de México sobre REDD+. Hacia una estrategia Nacional. México. 56 p.

Van Laar, A. y A. Akça. 2007. Forest Mensuration. Springer. Dordrecht. 383 p.

Manuscrito recibido el 27 de febrero de 2014.

Aceptado el 18 de septiembre de 2015.

Este documento se debe citar como:

Aguirre-Calderón, O.A. 2015. Manejo forestal en el siglo XXI. Madera y Bosques 21(Núm. esp.):17-28. 